Corrigendum

\title{
Corrigendum to "A Case of In-Bore Transperineal MRI-Guided Prostate Biopsy of a Patient with Ileal Pouch-Anal Anastomosis"
}

\author{
Michael Kongnyuy, ${ }^{1}$ Thomas Frye, ${ }^{1}$ Arvin K. George, ${ }^{1}$ \\ Amichai Kilchevsky, ${ }^{1}$ Amogh Iyer, ${ }^{1}$ Meet Kadakia, ${ }^{1}$ Akhil Muthigi, ${ }^{1}$ \\ Baris Turkbey, ${ }^{2}$ Bradford J. Wood, ${ }^{3}$ and Peter A. Pinto ${ }^{1,3}$ \\ ${ }^{1}$ Urologic Oncology Branch, National Cancer Institute, National Institute of Health, Bethesda, MD 20892, USA \\ ${ }^{2}$ Molecular Imaging Program, National Cancer Institute, National Institute of Health, Bethesda, MD 20892, USA \\ ${ }^{3}$ Center for Interventional Oncology, National Cancer Institute, National Institute of Health, Bethesda, MD 20892, USA \\ Correspondence should be addressed to Thomas Frye; thomas.frye@nih.gov \\ Received 7 April 2016; Accepted 19 April 2016
}

Copyright ( $) 2016$ Michael Kongnyuy et al. This is an open access article distributed under the Creative Commons Attribution License, which permits unrestricted use, distribution, and reproduction in any medium, provided the original work is properly cited.

In the article titled "A Case of In-Bore Transperineal MRIGuided Prostate Biopsy of a Patient with Ileal Pouch-Anal Anastomosis," [1] the name of the ninth author was given incorrectly as Brad J. Wood. The author's name should have been written Bradford J. Wood. The revised author list is shown above.

\section{References}

[1] M. Kongnyuy, T. Frye, A. K. George et al., "A case of in-bore transperineal MRI-guided prostate biopsy of a patient with ileal pouch-anal anastomosis," Case Reports in Urology, vol. 2015, Article ID 676930, 3 pages, 2015. 




The Scientific World Journal
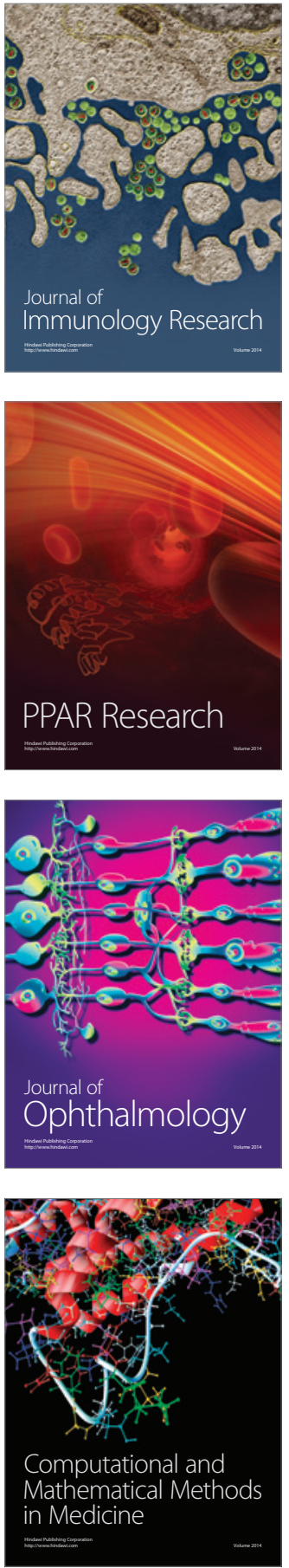

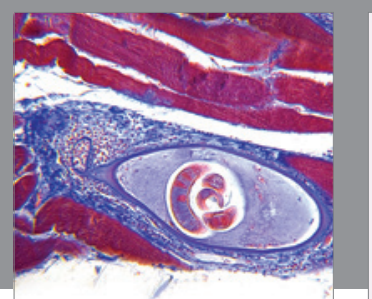

Gastroenterology Research and Practice

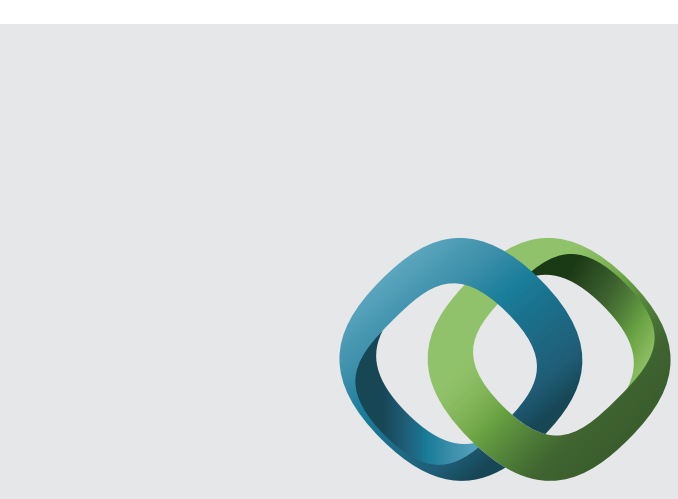

\section{Hindawi}

Submit your manuscripts at

http://www.hindawi.com
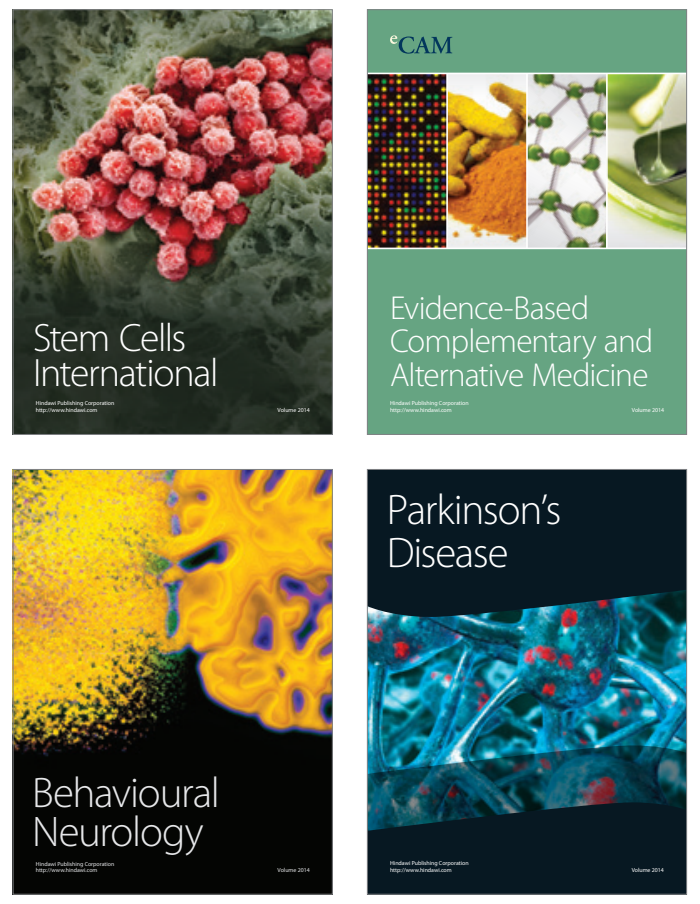
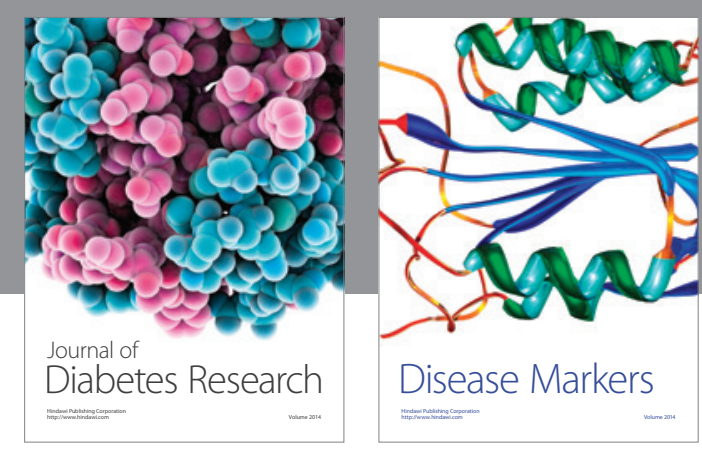

Disease Markers
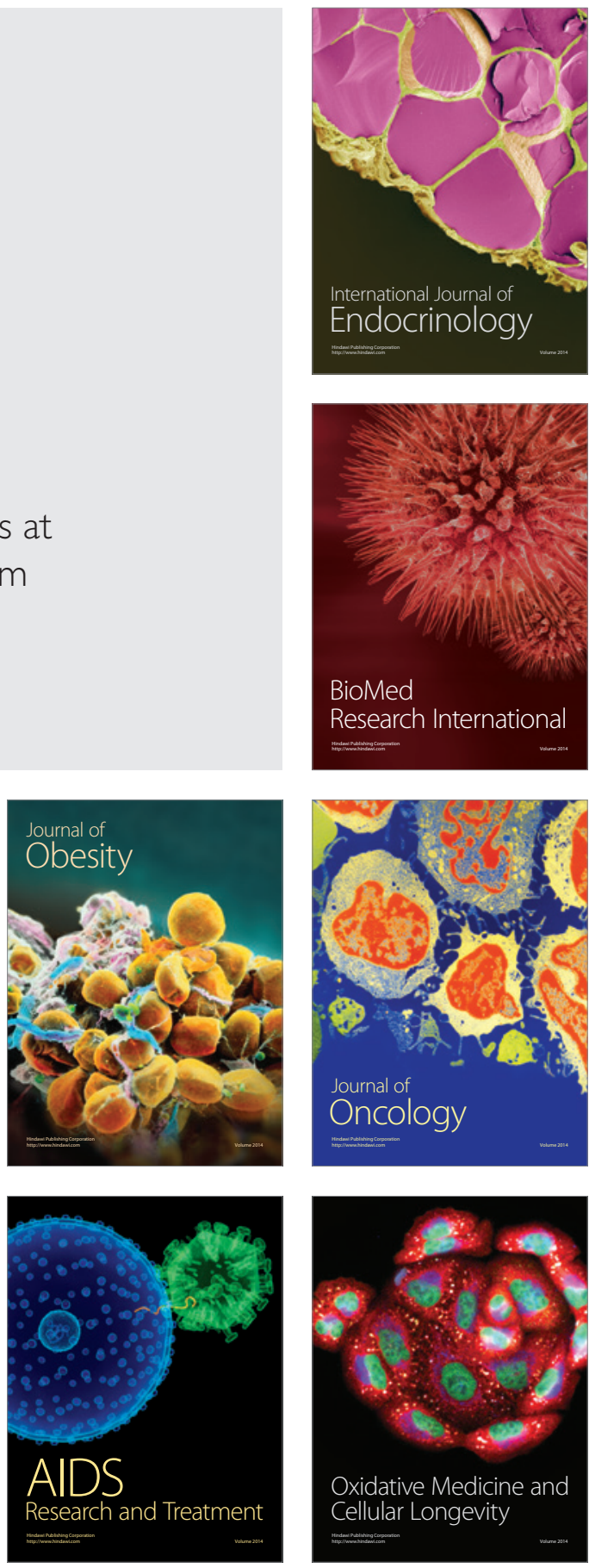